\title{
Good practices in free-energy calculations
}

\section{Andrew Pohorille. Christopher Jarzynski and Christophe Chipot}

As access to computational resources continues to increase, free-energy calculations have emerged as a powerful tool that can play a predictive role in drug design. Yet, in a number of instances, the reliability of these calculations can be improved significantly if a number of precepts, or good practices are followed. For the most part, the theory upon which these good practices rely has been known for many years, but often overlooked, or simply ignored. In other cases, the theoretical developments are too recent for their potential to be fully grasped and merged into popular platforms for the computation of free-energy differences. The current best practices for carrying out free-energy calculations will be reviewed demonstrating that, at little to no additional cost, freeenergy estimates could be markedly improved and bounded by meaningful error estimates. In energy perturbation and nonequilibrium work methods, monitoring the probability distributions that underlie the transformation between the states of interest, performing the calculation bidirectionally, stratifying the reaction pathway and choosing the most appropriate paradigms and algorithms for transforming between states offer significant gains in both accuracy and precision. In thermodynamic integration and probability distribution (histogramming) methods, properly designed adaptive techniques yield nearly uniform sampling of the relevant degrees of freedom and, by doing so, could markedly improve efficiency and accuracy of free energy calculations without incurring any additional computational expense. 